\title{
Clinical Study \\ Thyroid Nodules in Children: A Single Institution's Experience
}

\author{
Nini Khozeimeh and Cynthia Gingalewski \\ Department of Pediatric Surgery, Children's National Medical Center, 111 Michigan Avenue NW, Washington, DC 20010, USA \\ Correspondence should be addressed to Cynthia Gingalewski, cgingale@cnmc.org
}

Received 1 June 2011; Revised 11 August 2011; Accepted 13 August 2011

Academic Editor: David Ball

Copyright ( 12011 N. Khozeimeh and C. Gingalewski. This is an open access article distributed under the Creative Commons Attribution License, which permits unrestricted use, distribution, and reproduction in any medium, provided the original work is properly cited.

\begin{abstract}
Thyroid nodules in children are uncommon but often present an increased risk of malignancy in comparison to their adult counterpart. Multiple diagnostic modalities are frequently employed to characterize these nodules including ultrasound, radionuclide scans, fine needle aspiration (FNA), thyroid function tests, and evaluation of patient demographics. We chose to evaluate if any of these modalities influence treatment or signify a tendency for a nodule to represent a malignant lesion. A retrospective review of patients $<21$ years of age who underwent partial or total thyroidectomy from 2004 to 2009 was performed (IRB no. 4695). Other than an FNA indicating a malignancy, there does not appear to be any value to extensive preoperative imaging, nor can patient risk be stratified based upon age. We conclude that there is minimal utility in an extensive preoperative workup in a child with a thyroid nodule.
\end{abstract}

\section{Introduction}

Thyroid cancer is the most common endocrine malignancy in pediatric patients [1-3]. However, thyroid nodules in children are only found in $3.7 \%$ of healthy children aged $11-$ 18 years old [4]. Compared to adults, these nodules have an increased risk of being malignant ( $16 \%$ in children versus $5 \%$ in adults) [4-6]. Thyroid cancer in children is also unusual in that it often presents with advanced disease including lymph node involvement and lung metastasis as compared to their adult counterpart $[5,7-9]$.

Multiple modalities are frequently employed in an attempt to characterize thyroid nodules including ultrasound, radionuclide scans, fine needle aspiration (FNA), thyroid function tests, and evaluating patient demographics. In the adult population these results are frequently used to determine those patients who do not require thyroidectomy. Although these tests are also performed in the pediatric population, these patients frequently undergo partial or total thyroidectomy for diagnosis

The aim of this paper is to explore the utility of radiographic imaging and preoperative fine needle aspiration (FNA) in the evaluation of pediatric thyroid nodules, more specifically, to determine if any of these modalities identifies those thyroid nodules that are at high risk of harboring a malignancy.

\section{Methods}

A retrospective chart review was performed (2004-2009) at a tertiary medical center with a $100 \%$ pediatric patient population (IRB approval no. 4695). Fifty patients $<21$ years of age were identified who underwent partial or total thyroidectomy. Five patients with a family history of multiple endocrine neoplasia (MEN) were excluded, giving a total of 45 patients evaluated in the present study. Patient demographics, preoperative workup, type of procedure performed, and pathology results were reviewed. Calculations were performed using a 2-tailed Student's $t$-test.

\section{Results}

3.1. Demographics. Forty-five patients underwent either partial or total thyroidectomy; the majority of patients were female $(n=41)$. There was no difference in the median age at presentation for malignant and benign lesions 15 years (range of 7-19 years) versus 14 years (range of 8-21 years) $(P=.88)$, respectively. None of the patients identified had a history of previous neck irradiation. Patient demographics and tumor characteristics are summarized in Table 1.

3.2. Preoperative Workup. Ninety-one percent of patients underwent preoperative imaging with ultrasound $(n=31)$, CT $\operatorname{scan}(n=4), \mathrm{I}^{123} \operatorname{scan}(n=2)$, or ultrasound 
TABle 1: Demographic, tumor, and clinical characteristics. Thyroid nodules were identified as malignant or benign based on pathologic characteristics. These nodules were then characterized based on patient age, gender, and preoperative workup including imaging or FNA.

\begin{tabular}{lcc}
\hline & \multicolumn{2}{c}{ Entire cohort $n=45$} \\
& Malignant & Benign \\
\hline Total patients & 9 & 36 \\
Gender & 8 & 33 \\
$\quad$ Female & 1 & 3 \\
$\quad$ Male & 14.1 & 14.2 \\
Average age at diagnosis (years) & 2.7 & 2.9 \\
Average size of nodule (cm) & & \\
FNA & 5 & 17 \\
$\quad$ Yes & 4 & 19 \\
$\quad$ No & & \\
Radiologic imaging & 5 & 26 \\
$\quad$ Ultrasound & 3 & 2 \\
CT scan & 0 & 3 \\
$\quad$ Nuclear medicine & 1 & \\
$\quad$ Ultrasound and 2nd modality & & \\
\hline
\end{tabular}

and a second modality $(n=4)$. Of the 4 patients who had no preoperative imaging, three patients underwent thyroidectomy for a symptomatic, enlarging multinodular goiter. None of these patients were found to have malignancy. There were no herald radiologic findings to distinguish a lesion as malignant. Several nodules $(n=12)$ were found to have increased vascularity concerning malignancy; however, these nodules were found to be both malignant $(n=3)$ and benign $(n=9)$. FNA was performed in forty-nine percent of patients $(n=22)$. Of the nine patients found to have malignancy, five underwent preoperative FNA. Four FNAs were interpreted as malignant returning as papillary carcinoma. One FNA was interpreted as a follicular lesion but returned multifocal papillary carcinoma on final pathologic evaluation. This yields a sensitivity of $80 \%$ and specificity of $100 \%$.

3.3. Disease Characteristics. Nine out of forty-five patients were identified with papillary carcinoma on pathologic examination (20\%). Cervical lymph node involvement was identified in $56 \%$ percent of patients $(n=5)$ at the time of thyroidectomy. No patient had evidence of pulmonary metastases. There was no significant difference in the average size of the nodules in patients with malignant or benign disease $(2.7 \mathrm{~cm}$ and $2.9 \mathrm{~cm}$, resp., $P=.63)$.

3.4. Surgical Procedure. Total thyroidectomy was performed as the initial procedure in those patients identified with malignancy by FNA $(n=4)$. Two patients underwent completion thyroidectomy after pathologic examination of their thyroid lobe revealed papillary carcinoma. Both of these patients did not undergo preoperative FNA. Both patients underwent preoperative imaging; the first patient with an ultrasound that showed a vascular nodule, while the second patient had a CT scan that showed a small nodule. In addition, one patient was diagnosed with papillary carcinoma on an intraoperative frozen section and had a completion thyroidectomy at that time. One patient had an incidentally found micropapillary carcinoma in the setting of Grave's disease, and one patient with a multinodular goiter had a preoperative FNA showing follicular cells, and the final pathologic diagnosis was papillary carcinoma. These patients did have preoperative imaging that showed nodules.

Of those patients with benign disease $(n=36), 12(33 \%)$ underwent a total thyroidectomy for either an enlarging multinodular goiter or Grave's disease refractory to medical treatment. Twenty-four patients underwent lobectomy. Sixteen underwent a right lobectomy (45\%), and 8 had a left lobectomy (22\%).

Hypocalcemia was the most common postoperative complication, occurring in 8 patients $(38 \%)$. Half of these patients $(n=4)$ experienced symptoms with tingling or paresthesias, whereas the other half $(n=4)$ were identified with hypocalcemia on routine postoperative labs measurements. All patients were managed with supplemental oral calcium, and none experienced permanent hypocalcemia/hypoparathyroidism. All patients had normalization of their calcium levels within 3 months postoperatively. There were no injuries to the recurrent laryngeal nerve.

The average length of stay was similar for all patients after total thyroidectomy: 1.46 days for those with malignancy (range 1-5 days) and 1.53 days for benign pathology (range 1-8 days). All patients undergoing a thyroid lobectomy were discharged after a 23-hour observation period.

\section{Discussion}

Thyroid nodules in children represent an uncommon entity but carry a greater risk for malignancy than in their adult counterpart. In the pediatric population, an extensive preoperative workup of a thyroid nodule is not necessary. This should be limited to an ultrasound examination to determine that if indeed the nodule is within the thyroid gland. If feasible, given the patients age and anxiety level, a FNA should be performed. In those patients who have an inadequate FNA, or FNA is not performed, surgical excision should be performed for diagnosis. Total thyroidectomy remains the procedure of choice for those lesions identified preoperatively as cancer, while lobectomy should be employed for those lesions in which the diagnosis is uncertain [10-14]. This facilitates the use of $I^{131}$ therapy postoperatively as well as the ability to monitor thyroglobulin levels after treatment for recurrent disease. Overall, thyroid cancer has a very good prognosis with a $98.8 \%$ survival at 10 years in children $[2,7]$.

The female predominance of thyroid nodules noted in our cohort has been previously reported in the literature and is likely secondary to the estrogen sensitivity of the thyroid gland [15-18].

In our cohort, patient age and radiologic imaging bears no impact in determining whether a nodule was malignant or benign. Increased intranodular vascularity is an ultrasound characteristic that has been suggested as an indicator 
of malignancy $[19,20]$. In our group, patients were found to have intranodular vascularity suggestive of malignancy $(n=12)$ however, the lesions returned both benign $(n=9)$ and malignant $(n=3)$. Average nodule size also did not signify a predisposition for malignancy.

Fine needle aspiration and cytology evaluation in the pediatric patient is controversial [5, 21-25]. FNA is useful for preoperative planning if the lesion is identified as malignant. But the lesion may be identified as benign, indeterminate, or follicular. Follicular lesions cannot be differentiated as malignant or benign because of the inability of FNA to assess capsular invasion. If the lesion is identified as malignant, the patient should undergo a total thyroidectomy at initial operation versus an initial lobectomy when indeterminate or benign. If FNA identifies the nodule as benign or a cyst, a patient can be followed if the lesion is small and avoid operative intervention. In adults, the accuracy of FNA can reach $97 \%$, while the pediatric population accuracy only reaches $90 \%[5,8,21,23,26]$. Other limitations of FNA include sampling error, experience of the cytopathologist, or need for sedation in younger children.

Postoperative complications of hypocalcemia and recurrent laryngeal nerve injury are a great cause of concern in patients undergoing total thyroidectomy. The most common complication following total thyroidectomy is hypocalcemia. Hypocalcemia is largely due to incidental stunning or removal of the parathyroid glands, which may be embedded in the thyroid gland $[7,27]$. The parathyroid glands may also be devascularized during thyroid gland dissection. The resultant hypocalcemia can be either transient or permanent.

Permanent hypocalcemia was not encountered in our patient population. Another risk of total thyroidectomy is recurrent laryngeal nerve injury (RLN), resulting in hoarseness, dysphagia, and respiratory failure if a bilateral injury occurs. Permanent RLN after total thyroidectomy has been reported as $1 \%$ in the literature $[28,29]$. We report no instances of RLN injury in our group.

In our retrospective review, we report no instances of major complications, such as RLN injury or permanent hypocalcemia, following total thyroidectomy. Transient hypocalcemia is considered to be of moderate risk and is readily managed with oral calcium supplementation.

In conclusion, there does not seem to be any component found in the preoperative evaluation of a pediatric thyroid nodule, with the exception of a positive FNA to indicate that a lesion is malignant or benign. Our experience suggests that patients cannot be risk stratified based on age, size of the thyroid lesion, or characteristics of the lesion by all radiographic imaging techniques. In addition, postoperative risk to patients undergoing lobectomy or total thyroidectomy remains low and predominantly consists of transient hypocalcemia. At our institution we have found minimal utility of an extensive preoperative workup in a child with a thyroid nodule. CT scans have not been shown to be sensitive or specific for determining whether a lesion is cancerous or benign. Therefore, the lifelong risk of cancer associated with CT is not justified (lifetime cancer risk associated with CT is estimated to be $2 / 1000$ to $3 / 1000$ in children under age 15). We advocate ultrasound evaluation to determine an intrathyroidal location, an FNA if feasible, and then either total thyroidectomy for those with malignancy identified on cytology or lobectomy for those whose diagnosis is uncertain.

\section{References}

[1] C. A. Dinauer, C. Breuer, and S. A. Rivkees, "Differentiated thyroid cancer in children: diagnosis and management," Current Opinion in Oncology, vol. 20, no. 1, pp. 59-65, 2008.

[2] M. T. Parisi and D. Mankoff, "Differentiated pediatric thyroid cancer: correlates with adult disease, controversies in treatment," Seminars in Nuclear Medicine, vol. 37, no. 5, pp. 340356, 2007.

[3] L. Ries, D. Melbert, M. Krapcho et al., "SEER Cancer Statistics Review," 2008.

[4] M. L. Rallison, B. M. Dobyns, A. W. Meikle, M. Bishop, J. L. Lyon, and W. Stevens, "Natural history of thyroid abnormalities: prevalence, incidence, and regression of thyroid diseases in adolescents and young adults," American Journal of Medicine, vol. 91, no. 4, pp. 363-370, 1991.

[5] S. S. Raab, J. F. Silverman, T. M. Elsheikh, P. A. Thomas, and P. E. Wakely, "Pediatric thyroid nodules: disease demographics and clinical management as determined by fine needle aspiration biopsy," Pediatrics, vol. 95, no. 1, pp. 46-49, 1995.

[6] J. I. Cohen and K. D. Salter, "Thyroid disorders: evaluation and management of thyroid nodules," Oral and Maxillofacial Surgery Clinics of North America, vol. 20, no. 3, pp. 431-443, 2008.

[7] Y. E. Demidchik, E. P. Demidchik, C. Reiners et al., "Comprehensive clinical assessment of 740 cases of surgically treated thyroid cancer in children of Belarus," Annals of Surgery, vol. 243, no. 4, pp. 525-532, 2006.

[8] A. Al-Shaikh, B. Ngan, A. Daneman, and D. Daneman, "Fine-needle aspiration biopsy in the management of thyroid nodules in children and adolescents," Journal of Pediatrics, vol. 138, no. 1, pp. 140-142, 2001.

[9] C. A. Gingalewski and K. D. Newman, "Seminars: controversies in the management of pediatric thyroid malignancy," Journal of Surgical Oncology, vol. 94, no. 8, pp. 748-752, 2006.

[10] P. W. Grigsby, A. Gal-or, J. M. Michalski, and G. M. Doherty, "Childhood and adolescent thyroid carcinoma," Cancer, vol. 95, no. 4, pp. 724-729, 2002.

[11] I. Demidchik and V. A. Kontratovich, "Repeat surgery for recurrent thyroid carcinoma in children," Voprosy Onkologii, vol. 49, no. 3, pp. 366-369, 2003.

[12] D. Handkiewicz-Junak, J. Wloch, J. Roskosz et al., "Total thyroidectomy and adjuvant radioiodine treatment independently decrease locoregional recurrence risk in childhood and adolescent differentiated thyroid cancer," Journal of Nuclear Medicine, vol. 48, no. 6, pp. 879-888, 2007.

[13] H. Gharib and E. Papini, "Thyroid nodules: clinical importance, assessment, and treatment," Endocrinology and Metabolism Clinics of North America, vol. 36, no. 3, pp. 707735, 2007.

[14] C. Spinelli, A. Bertocchini, A. Antonelli, and P. Miccoli, "Surgical therapy of the thyroid papillary carcinoma in children: experience with 56 patients < or = 16 years old," Journal of Pediatric Surgery, vol. 39, no. 10, pp. 1500-1505, 2004.

[15] K. D. Newman, T. Black, G. Heller et al., "Differentiated thyroid cancer: determinants of disease progression in patients $<21$ years of age at diagnosis: a report from the surgical 
discipline committee of the children's cancer group," Annals of Surgery, vol. 227, no. 4, pp. 533-541, 1998.

[16] N. L. Shapiro and N. Bhattacharyya, "Population-based outcomes for pediatric thyroid carcinoma," Laryngoscope, vol. 115, no. 2, pp. 337-340, 2005.

[17] K. W. Gow, S. Lensing, D. A. Hill et al., "Thyroid carcinoma presenting in childhood or after treatment of childhood malignancies: an institutional experience and review of the literature," Journal of Pediatric Surgery, vol. 38, no. 11, pp. 1574-1580, 2003.

[18] Y. Imai, M. Yamakawa, M. Matsuda, and T. Kasajima, "Endogenous sex hormone and estrogen binding activity in thyroid cancer," Histology and Histopathology, vol. 4, no. 1, pp. 39-45, 1989.

[19] T. Rago and P. Vitti, "Role of thyroid ultrasound in the diagnostic evaluation of thyroid nodules," Best Practice \& Research Clinical Endocrinology \& Metabolism, vol. 22, no. 6, pp. 913-928, 2008.

[20] S. Bastin, M. J. Bolland, and M. S. Croxson, "Role of ultrasound in the assessment of nodular thyroid disease," Journal of Medical Imaging and Radiation Oncology, vol. 53, no. 2, pp. 177-187, 2009.

[21] H. Gharib, "Fine-needle aspiration biopsy of thyroid nodules: advantages, limitations, and effect," Mayo Clinic Proceedings, vol. 69, no. 1, pp. 44-49, 1994.

[22] F. W. Yip, T. S. Reeve, A. G. Poole, and L. Delbridge, “Thyroid nodules in childhood and adolescence," Australian and New Zealand Journal of Surgery, vol. 64, no. 10, pp. 676-678, 1994.

[23] E. L. Mazzaferri, "Management of a solitary thyroid nodule," The New England Journal of Medicine, vol. 328, no. 8, pp. 553 559, 1993.

[24] H. Lugo-Vicente, V. N. Ortíz, H. Irizarry, J. I. Camps, and V. Pagán, "Pediatric thyroid nodules: management in the era of fine needle aspiration," Journal of Pediatric Surgery, vol. 33, no. 8, pp. 1302-1305, 1998.

[25] M. Amrikachi, T. B. Ponder, T. M. Wheeler, D. Smith, and I. Ramzy, "Thyroid fine-needle aspiration biopsy in children and adolescents: experience with 218 aspirates," Diagnostic Cytopathology, vol. 32, no. 4, pp. 189-192, 2005.

[26] S. Corrias, E. Einaudi, G. Chiorboli et al., "Accuracy of fine needle aspiration biopsy of thyroid nodules in detecting malignancy in children: comparison with conventional clinical, laboratory, and imaging approaches," The Journal of Clinical Endocrinology \& Metabolism, vol. 8, pp. 4644-4648, 2001.

[27] L. Rosato, N. Avenia, P. Bernante et al., "Complications of thyroid surgery: analysis of a multicentric study on 14,934 patients operated on in Italy over 5 Years," World Journal of Surgery, vol. 28, no. 3, pp. 271-276, 2004.

[28] M. P. La Quaglia, T. Black, G. W. Holcomb III et al., "Differentiated thyroid cancer: clinical characteristics, treatment, and outcome in patients under 21 years of age who present with distant metastases. A report from the surgical discipline committee of the children's cancer group," Journal of Pediatric Surgery, vol. 35, no. 6, pp. 955-960, 2000.

[29] T. Reeve and N. W. Thompson, "Complications of thyroid surgery: how to avoid them, how to manage them, and observations on their possible effect on the whole patient," World Journal of Surgery, vol. 24, no. 8, pp. 971-975, 2000. 


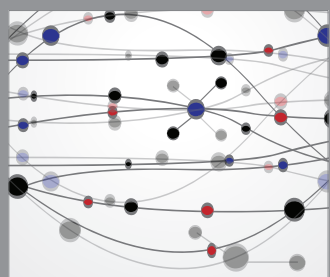

The Scientific World Journal
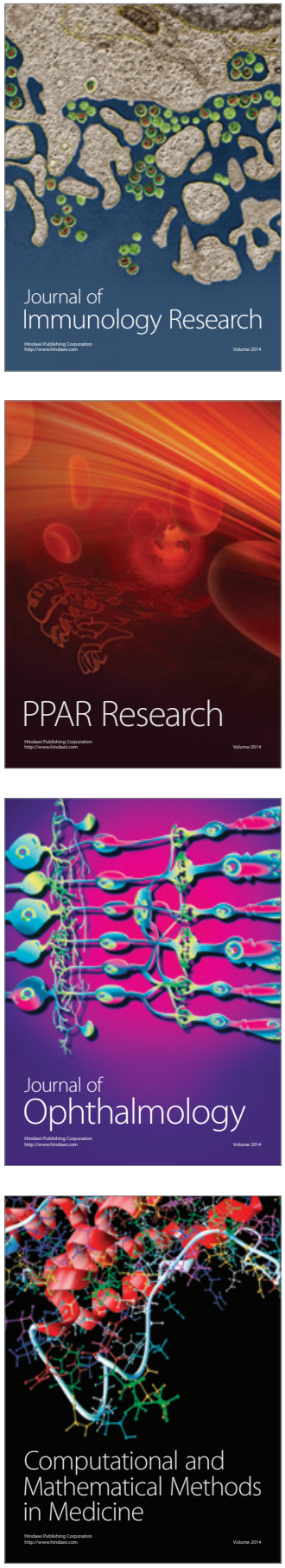

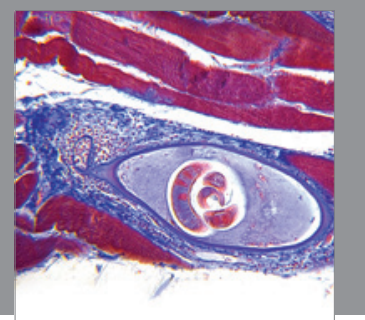

Gastroenterology

Research and Practice
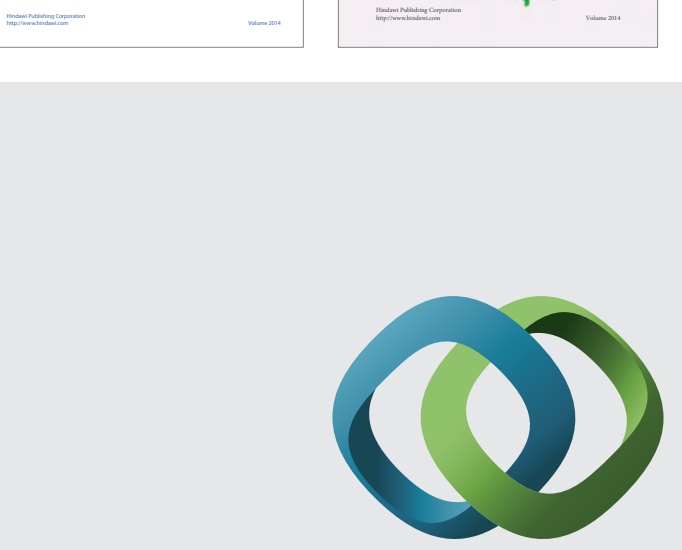

\section{Hindawi}

Submit your manuscripts at

http://www.hindawi.com
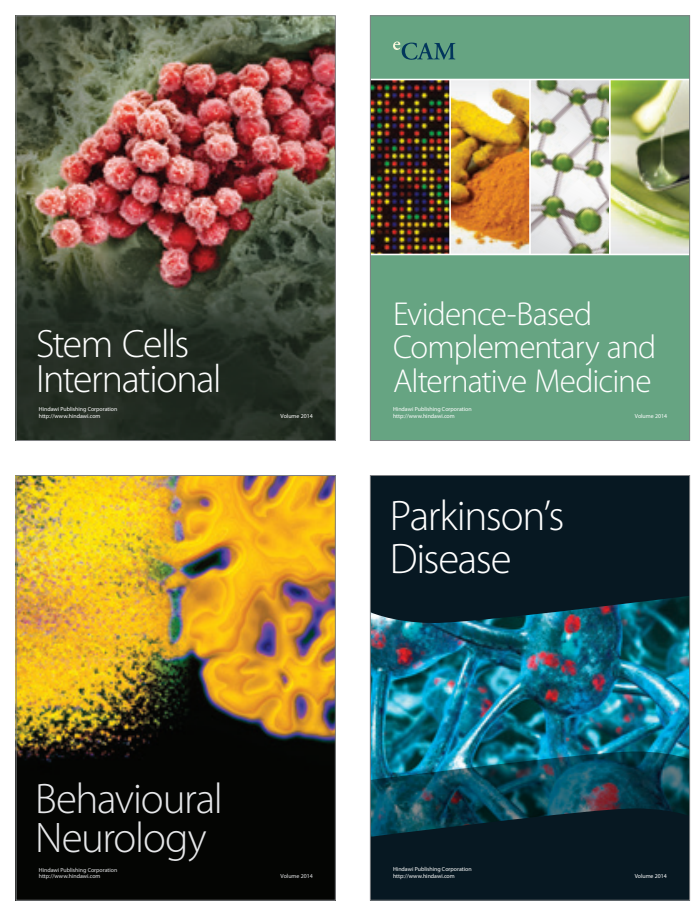

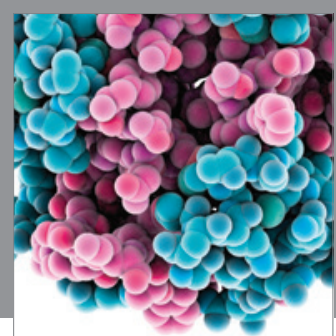

Journal of
Diabetes Research

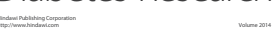

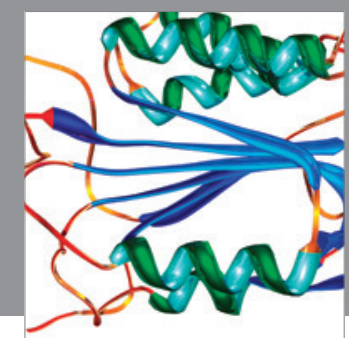

Disease Markers
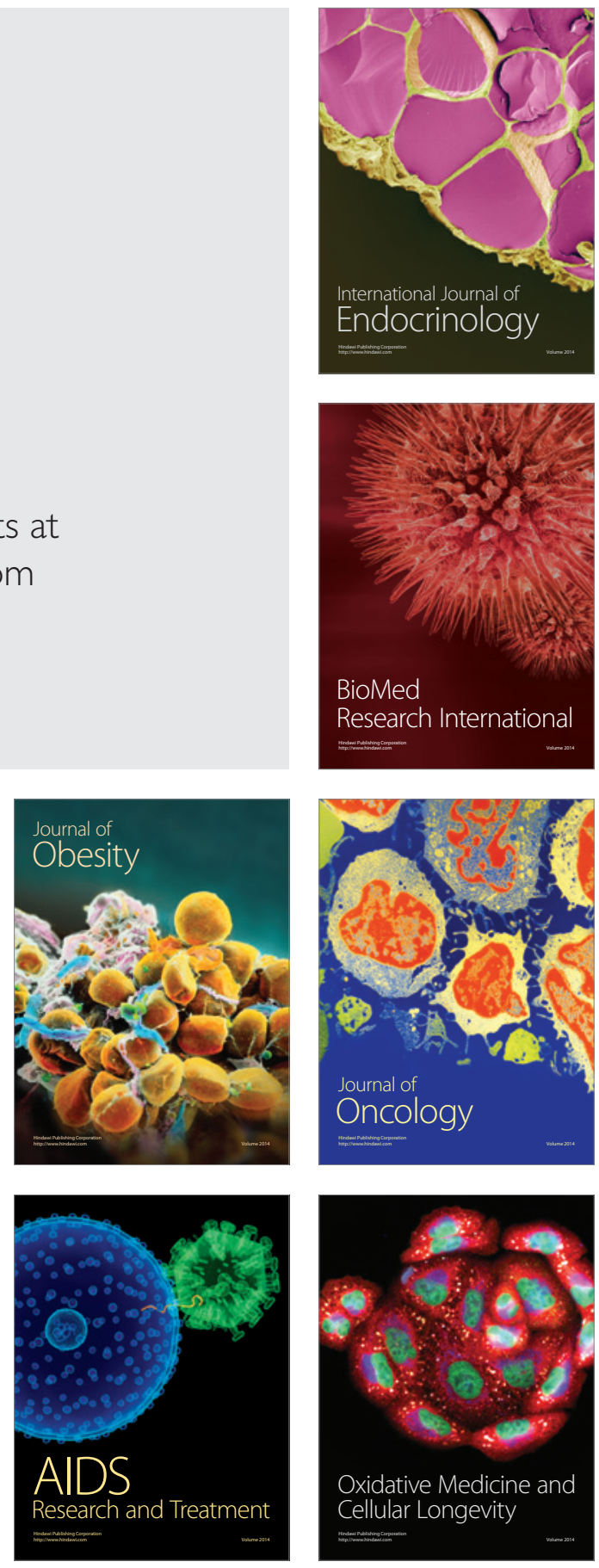